\title{
Manejo anestésico en paciente con miocardiopatía periparto
}

\author{
Anesthetic management in patient with peripartum cardiomyopathy \\ Mariela García Bravo, * Idaleyvis German Córdoba, ${ }^{\ddagger}$ \\ José Manuel Athié García, ${ }^{\S}$ Eric Misael Saucedo Moreno
}

Citar como: García BM, German Cl, Athié GJM, Saucedo MEM. Manejo anestésico en paciente con miocardiopatía periparto. Acta Med. 2020; 18 (4): 395-398. https://dx.doi.org/10.35366/97266

\section{Resumen}

Con el objetivo de describir la fisiopatología de la miocardiopatía periparto, una enfermedad de baja incidencia y la importancia del manejo anestésico que estas pacientes requieren, se realizó la siguiente revisión bibliográfica. Se llegó a la conclusión de que a pesar de ser una enfermedad de rara presentación, tiene alta mortalidad en mujeres embarazadas, en quienes es recomendable el tratamiento con heparina de bajo peso molecular por la teratogenia que pueden producir otros anitcoagulantes al igual que el manejo de primera línea requerido. El manejo anestésico es de suma importancia para el mantenimiento adecuado tanto de la precarga como de la postcarga así como la determinación del tipo de anestesia que se le puede ofrecer a la paciente teniendo en cuenta estudios de laboratorio para poder elegir técnicas regionales que en estos casos es lo ideal. Se debe estar preparado para cualquier manejo y tener al alcance los coadyuvantes necesarios para mejorar y mantener un adecuado estado hemodinámico.

Palabras clave: Miocardiopatía, periparto, embarazo, fisiopatología, anestesia, monitoreo.

\section{INTRODUCCIÓN}

La miocardiopatía periparto (MCPP) es una entidad clínica que se presenta en raras ocasiones, es potencialmente mortal y de etiología desconocida.

\section{Abstract}

In order to describe the pathophysiology of peripartum cardiomyophaty -a low incidence disease- and the importance of anesthetic management, these patients underwent the following bibliography review. It was concluded that despite being a rare disease, it has high mortality in pregnant women who should be treated with low molecular weight heparin due to the incidence of teratogenicity with other anticoagulants, as well as the first line treatment needed. The anesthetic management is crucial to maintain an adequate preload and afterload, as well as the determination of the type of anesthesia that can be offered to the patient taking into account the laboratory studies to be able to choose regional techniques -which in these cases, is ideal-Being prepared to handle any complications and have the necessary adjuvants available to improve and maintain an adequate hemodynamic state.

Keywords: Cardiomiopathy, peripartum, pregnancy, pathophysiology, anesthesia, management.

Se define según la Heart Failure Association of the European Society of Cardiology Working Group on PPCM como "una insuficiencia cardiaca secundaria a disfunción sistólica del ventrículo izquierdo (VI) en el tercer trimestre del embarazo o en los cinco meses posteriores al parto,

* Residente de tercer año de Anestesiología. Hospital ABC Observatorio. Universidad Nacional Autónoma de México.

‡ Residente de tercer año de Anestesiología. Facultad Mexicana de Medicina. Universidad La Salle México.

§ Médico Anestesiólogo. Director Médico. Profesor Titular de la Especialidad de Anestesiología.

" Cirujano General adscrito al Servicio de Cirugía General.

Correspondencia:

Idaleyvis German Córdoba

Correo electrónico: idaleyvis@hotmail.com

Aceptado: 25-03-2020.

www.medigraphic.com/actamedica

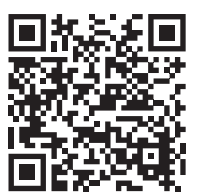


donde no se encuentra otra causa de insuficiencia cardiaca. Es un diagnóstico de exclusión. El VI puede no dilatarse, pero la fracción de eyección casi siempre se reduce por debajo de $45 \%{ }^{\prime \prime}{ }^{1}$

Es una entidad rara que afecta $0.1 \%$ de todos los embarazos, tiene alta mortalidad y morbilidad de hasta $1 / 3 .^{2,3}$

\section{FISIOPATOLOGÍA Y FACTORES DE RIESGO}

La etiología de esta patología se desconoce, se cree que se encuentran implicados factores inmunológicos que causan sensibilización contra antígenos de células hematopoyéticas fetales. Existen reportes de autores que la consideran una miocardiopatía de origen genético-familiar desencadenada por el estrés hemodinámico que supone la gestación.

Existen factores de riesgo que se han visto relacionados con la enfermedad como la edad materna tardía, la multiparidad, los embarazos múltiples, la raza afroamericana o los trastornos hipertensivos durante el embarazo que también parecen propiciar esta complicación. ${ }^{4}$

\section{CUADRO CLÍNICO}

Se presenta con insuficiencia cardiaca (IC) secundaria a la disfunción sistólica del VI al final del embarazo o en los cinco meses posteriores al parto. Es un diagnóstico de exclusión cuando no se encuentra otra causa para la IC aguda. ${ }^{5}$

Los signos y síntomas suelen ser típicos de IC, pero se describe un amplio espectro de síntomas en pacientes con MCPP. Se han descrito arritmias ventriculares complejas y parada cardiaca súbita. ${ }^{6}$

\section{PRONÓSTICO}

El pronóstico de la MCPP se divide en el pronóstico materno y obstétrico. La mortalidad materna va de 6 a $60 \%$, principalmente debido a una falla de bomba progresiva y a eventos tromboembólicos. ${ }^{7}$ Se ha observado también que la mortalidad es más alta si la paciente inicia con peor capacidad funcional y es multípara. ${ }^{8}$ Respecto al pronóstico obstétrico, $25 \%$ de las pacientes tienen tendencia a un parto prematuro y $40 \%$ a partos por vía cesárea. La posibilidad de embarazos a futuro tiene que ver principalmente con la severidad con que se presentó la miocardiopatía periparto (MP) y del grado de disfunción ventricular izquierda persistente con el que quedó la madre, pero en general se puede decir que las mujeres que presentan una MP tienen mayor riesgo en embarazos posteriores. ${ }^{9}$

\section{TRATAMIENTO}

Los objetivos del tratamiento médico en pacientes con diagnóstico MCPP deben incluir medidas para mejorar la oxigenación y mantener el gasto cardiaco, al igual que el resultado materno y fetal. Se requieren intervenciones para disminuir tanto la precarga como la postcarga así como para mejorar la contractilidad cardiaca. ${ }^{10}$

La primera línea de terapia incluye diuréticos, vasodilatadores acompañados de diuréticos y restricción de sodio. Después del parto, la paciente puede cambiarse a otras modalidades disponibles como inhibidores de la enzima convertidora de angiotensina, bloqueo beta y anticoagulación. Recientemente se ha demostrado que el selenio pentoxifilina y las inmunoglobulinas tienen un efecto beneficioso. Sin embargo, si estas modalidades no mejoran la sintomatología, puede indicarse el trasplante cardiaco. ${ }^{10}$

Se recomienda la anticoagulación en pacientes con MMCP, especialmente si la fracción de eyección es inferior a $35 \%$ y hay otros factores de riesgo asociados como ventrículos severamente dilatados, fibrilación auricular y presencia de trombo mural en la ecocardiografía o antecedentes sugestivos de episodios tromboembólicos anteriores. ${ }^{11} \mathrm{La}$ warfarina es teratogénica al principio del embarazo y puede causar el síndrome de warfarina fetal, mientras que la ingesta en el segundo y tercer trimestre puede provocar hemorragia cerebral fetal, microcefalia, ceguera, sordera y retraso del crecimiento. La heparina no fraccionada, por otro lado, tiene una baja biodisponibilidad en pacientes embarazadas y está asociada con trombocitopenia. Por lo tanto, se prefieren las heparinas de bajo peso molecular en el embarazo, ya que no cruzan la placenta, tienen menor riesgo de osteoporosis y trombocitopenia y su biodisponibilidad es más predecible. ${ }^{12}$

\section{EVALUACIÓN PREANESTÉSICA}

La presencia de esta condición plantea desafíos adicionales para el anestesiólogo. El más importante es realizar un diagnóstico correcto, ya que los síntomas de insuficiencia cardiaca como disnea, fatiga y edema de pedal pueden simplemente transmitirse debido a cambios fisiológicos normales en un parto.

Se debe optimizar a las pacientes y elegir la técnica anestésica que esté más acorde a sus condiciones hemodinámicas, tratamiento médico de base, anticoagulación, parto o cesárea y en caso de esta última, si es electiva o de urgencia.

Los objetivos de manejo anestésico son: ${ }^{13}$

- Mantener la perfusión miocárdica.

- Evitar: arritmias, episodios de hipotensión o taquicardia.

- Optimizar el gasto cardiaco.

- Mantener la precarga, pero evitar la sobrecarga de líquidos.

- Mantener o aumentar la contractilidad miocárdica.

- Evitar el aumento de la postcarga. 
Existen distintos índices de riesgo cardiaco perioperatorio en pacientes cardiópatas sujetos a cirugía no cardiaca. Para predecir el pronóstico de las pacientes embarazadas con cardiopatías se ha utilizado la clasificación funcional de la New York Heart Association (NYHA) (Tabla 1). ${ }^{14}$

Monitoreo: el monitoreo laboral estándar incluye la medición no invasiva de la presión arterial (NIBP), la tocodinamometría y el monitoreo de la frecuencia cardiaca fetal (FC). En pacientes con enfermedad cardiovascular de alto riesgo, se recomienda un monitoreo materno continuo adicional. Se puede justificar la canalización venosa y arterial central. ${ }^{15,16} \mathrm{El}$ uso perioperatorio de catéter de arteria pulmonar y ecocardiografía transesofágica se ha descrito en pacientes con función cardiaca severamente deprimida. ${ }^{17,18}$

La ventilación no invasiva con presión espiratoria final positiva adecuada puede instituirse si el oxígeno mediante una máscara facial simple no mejora la saturación de oxígeno en más de 95\%. En caso de que se requiera ventilación invasiva, se deben tomar precauciones estándar con respecto a la posibilidad de aspiración en una paciente embarazada. ${ }^{19}$

La técnica anestésica óptima para pacientes con MCPP programadas para someterse a cesárea es controvertida, ya que tanto la anestesia general como la regional se han descrito con éxito. ${ }^{19}$

El dolor y la ansiedad asociados con el trabajo de parto pueden aumentar la actividad del sistema nervioso simpático con un incremento resultante en el gasto cardiaco y la resistencia vascular periférica que conduce a un aumento en la postcarga cardiaca. Esta actividad simpática también disminuye el flujo de salida uteroplacentario, poniendo en peligro al feto ya comprometido. La analgesia efectiva en el trabajo de parto disminuye el flujo simpático y baja los niveles plasmáticos de catecolaminas en la madre. ${ }^{20}$ Se pueden proporcionar múltiples modalidades de analgesia laboral a estas pacientes para prevenir el aumento de la postcarga cardiaca asociada con dolores de parto, pero la anestesia regional (AR) sigue siendo el método de elección, ya que la simpatectomía asociada con ella causa una disminución de la precarga y postcarga cardiaca que es beneficiosa en pacientes con MMCP. ${ }^{21}$ Schnaider y colaboradores describen anestesia combinada epidural-espinal en una paciente obesa mórbida con una fracción de eyección de $20 \%$ que se sometió a cesárea con éxito bajo AR y tuvo un curso perioperatorio sin incidentes. ${ }^{22}$ El uso de un catéter brinda libertad de titulación del anestésico local tanto en el espacio epidural como intratecal. ${ }^{23}$ Por lo tanto, el nivel de bloqueo motor y sensorial se puede aumentar gradualmente para no causar hipotensión repentina que puede conducir a una descompensación repentina en este tipo de pacientes. ${ }^{24}$ La AR, sin embargo, puede estar contraindicada en una paciente anticoagulada.

La segunda etapa del trabajo de parto durante el parto vaginal debe reducirse mediante la aplicación de fórceps o aspiradora para acelerar el parto del bebé. Es importante evitar cualquier sobrecarga de líquidos durante el parto y el postparto. ${ }^{19}$

La anestesia general puede usarse para una cesárea urgente. ${ }^{5}$ Se debe recordar que las pacientes deberán ser manejadas con extrema precaución por el riesgo de regurgitación. El estrés de la laringoscopia y la intubación junto con los efectos depresores miocárdicos de los anestésicos no son beneficiosos para estas pacientes. Se debe inducir a la paciente con medicamentos que no ocasionen demasiada cardiodepresión. La anestesia a base de opioides proporciona un buen control hemodinámico y la respuesta de la intubación endotraqueal. ${ }^{19}$

Estas pacientes pueden necesitar optimización con agentes inotrópicos como la dobutamina o la milrinona. La respuesta de estos agentes debe monitorearse. ${ }^{19} \mathrm{El}$ levosimendán es otro agente cardiotrópico novedoso que mejora el gasto cardiaco al aumentar la respuesta de los miofilamentos al calcio intracelular, a diferencia de los inotrópicos tradicionales mencionados anteriormente que

Tabla 1: Clasificación funcional NYHA.

Clase

I Sin limitación de la actividad física. La actividad ordinaria no ocasiona excesiva fatiga, palpitaciones, disnea o dolor anginoso

II Ligera limitación de la actividad física. Confortables en reposo. La actividad ordinaria ocasiona fatiga, palpitaciones, disnea o dolor anginoso

III Marcada limitación de la actividad física. Confortables en reposo. Actividad física menor que la ordinaria ocasiona fatiga, palpitaciones, disnea o dolor anginoso

IV Incapacidad para llevar a cabo cualquier actividad física sin molestias. Los síntomas de insuficiencia cardiaca o de síndrome anginoso pueden estar presentes incluso en reposo. Si se realiza cualquier actividad física, el malestar aumenta 
aumentan el calcio intracelular mismo. Se ha demostrado que el levosimendán es eficaz para mejorar el gasto cardiaco y disminuir la mortalidad en pacientes con insuficiencia cardiaca severamente baja; ${ }^{23,24}$ sin embargo, su seguridad y eficacia en MMCP no han sido evaluadas por estudios controlados aleatorios, aunque existen informes de su uso exitoso en esta condición en la literatura. ${ }^{24}$ Levosimendán se usa como una infusión intravenosa a razón de 0.1-0.2 $\mu \mathrm{g} / \mathrm{kg} / \mathrm{min}$ en insuficiencia cardiaca con o sin una dosis de carga de 3-12 $\mu \mathrm{g} / \mathrm{kg}$ durante 10 minutos. ${ }^{25}$

En pacientes con presión arterial sistólica superior a $110 \mathrm{mmHg}$, la nitroglicerina (NTG) puede ser una opción. Se debe administrar mediante infusión intravenosa. Sin embargo, el nitroprusiato puede estar relativamente contraindicado en pacientes embarazadas debido al riesgo de acumulación de tiocianato y cianuro en el feto. La NTG debe titularse para que comience a partir de una dosis de $10-20 \mu \mathrm{g} / \mathrm{min}$ hasta un máximo de $200 \mu \mathrm{g} / \mathrm{min}^{19}$

Estas pacientes deben tener un seguimiento regular con cardiólogos para la evaluación continua de la función cardiaca.

\section{REFERENCIAS}

1. Sliwa K, Hilfiker-Kleiner D, Petrie MC, Mebazaa A, Pieske B, Buchmann E, McMurray JJ. Current state of knowledge on aetiology, diagnosis, management, and therapy of peripartum cardiomyopathy: a position statement from the Heart Failure Association of the European Society of Cardiology Working Group on peripartum cardiomyopathy. Eur J Heart Fail. 2010; 12 (8): 767-778.

2. Johnson-Coyle L, Jensen L, Sobey A. Peripartum cardiomyopathy: review and practice guidelines. Am J Crit Care. 2012; 21 (2): 89-98.

3. Tidswell M. Peripartum cardiomyopathy. Crit Care Clin. 2004; 20 (2): 777-788.

4. Elkayam U, Goland S, Pieper PG, Silversides CK. High-risk cardiac disease in pregnancy. J Am Coll Cardiol. 2016; 68 (5): 502-516.

5. Ware JS, Li J, Mazaika E, Yasso CM, DeSouza T, Cappola TP et al. Shared genetic predisposition in peripartum and dilated cardiomyopathies. NEJM. 2016; 374 (3): 233-241.

6. Haghikia A, Podewski E, Libhaber E, Labidi S, Fischer D, Roentgen P et al. Phenotyping and outcome on contemporary management in a German cohort of patients with peripartum cardiomyopathy. Basic Res Cardiol. 2013; 108 (4): 366.

7. Felker GM, Thompson RE, Hare JM, Hruban RH, Clemetson DE, Howard DL et al. Underlying causes and long-term survival in patients with initially unexplained cardiomyopathy. NEJM. 2000; 342 (15): 1077-1084.
8. Murali S, Baldisseri MR. Peripartum cardiomyopathy. Crit Care Med. 2005; 33 (10 Suppl): S340-346.

9. Sliwa K, Forster O, Zhanje F, Candy G, Kachope J, Essop R. Outcome of subsequent pregnancy in patients with documented peripartum cardiomyopathy. Am J Cardiol. 2004; 93 (11): 1441-1443.

10. Regitz-Zagrosek V, Roos-Hesselink JW, Bauersachs J, BlomströmLundqvist C, Cífková R, De Bonis M, Nelson-Piercy C. ESC Guidelines for the management of cardiovascular diseases during pregnancy. Eur Heart J. 2018; 39 (34): 3165-3241.

11. James $A H$, Jamison MG, Brancazio LR, Myers ER. Venous thromboembolism during pregnancy and the postpartum period: Incidence, risk factors, and mortality. Am J Obstet Gynecol. 2006; 194 (5): 1311-1315.

12. Davis SM, Branch DW. Thromboprophylaxis in pregnancy: who and how? Obst Gynecol Clin N Am. 2010; 37 (2): 333-343.

13. Hughes S. The obstetric patient with cardiac disease. In: Collins RE, Plaat F, Urguhart J. Textbook of obstetric Anaesthesia. London: Greenwich Medical Media. 2002, pp. 177-201.

14. Martín J, Carvajal A, Arantzamendi M. Instrumentos para valorar al paciente con insuficiencia cardiaca avanzada: una revisión de la literatura. Anales Sis San Navarra. 2015; 38(3): 439-452.

15. Soni B, Gautam PL, Grewal A, Kaur H. Anaesthetic management of two cases of peripartum cardiomyopathy. J Obstet Anaesth Crit Care. 2011; (1): 41-45.

16. Patil V. Anaesthetic management in peripartum cardiomyopathy. J Anest \& Inten Care Med. 2018; 6 (1): 1-4.

17. Velickovic I, Leicht C. Continuous spinal anesthesia for cesarean section in a parturient with severe recurrent peripartum cardiomyopathy. Int J of Obstet Anesth. 2004; 13 (1): 40-43.

18. Pryn A, Bryden F, Reeve W, Young S, Patrick A, McGrady E. Cardiomyopathy in pregnancy and caesarean section: Four case reports. Int J of Obstet Anesth. 2007; 16 (1): 68-73.

19. Arendt, K, Lindley K. Obstetric anesthesia management of the patient with cardiac disease. Int J of Obstet Anesth. 2019; 37: 73-85.

20. Ray P, Murphy G, Shutt L. Recognition and management of maternal cardiac disease in pregnancy. BJA. 2004; 93 (3): 428-439.

21. PyattJR, Dubey G. Peripartum cardiomyopathy: current understanding, comprehensive management review and new developments. Postgrad Med J. 2010; 87 (1023): 34-39.

22. Shnaider R, Ezri T, Szmuk P, Larson S, Warters RD, Katz J. Combined spinal-epidural anesthesia for Cesarean section in a patient with peripartum dilated cardiomyopathy. Can J Anesth/J Can Anesth. 2001; 48 (7): 681-683.

23. Tiwari A, Agrawal J, Tayal S, Chadha M, Singla A, Valson G et al. Anesthetic management of peripartum cardiomyopathy using "epidural volume extension" technique: a case series. Ann Card Anaesth. 2012; 15 (1): 44.

24. Castaño SJ, Castillo MJ, Escolano VF, Gallar GL, Montes PA, Samsó SE. Anestesia y analgesia obstétricas. Madrid: Ergon. 2007, pp. 125-134.

25. Mebazaa A, Nieminen MS, Packer M, Cohen-Solal A, Kleber FX, Pocock SJ et al. Levosimendan vs dobutamine for patients with acute decompensated heart failure. JAMA. 2007; 297 (17): 1883-1891. 